$\mathrm{DTP} / 96 / 04$

January 1996

\title{
Non-minimal neutral Higgs bosons at LEP2
}

\author{
A.G. Akeroyd目 \\ Department of Mathematical Sciences, Centre for Particle Theory, \\ University of Durham, \\ Durham DH1 3LE, England.
}

\begin{abstract}
We study the phenomenology of the neutral Higgs sector of a non-SUSY non-minimal Standard Model. Models with more than one Higgs doublet are possible, and may contain neutral Higgs scalars with branching ratios significantly different to those of the Minimal Standard Model Higgs boson. We show how these differences may be exploited at LEP2 in order to distinguish the non-minimal Standard Model from the minimal version.
\end{abstract}

\footnotetext{
${ }^{1}$ A.G.Akeroyd@durham.ac.uk
} 


\section{Introduction}

It is well known that the Standard Model (SM) [1] requires the breaking of the $S U(2) \times U(1)$ symmetry. Introducing a complex scalar doublet with a non-zero vacuum expectation value (VEV) is an elegant way of achieving this, and predicts one neutral scalar - the Higgs boson $\left(\phi^{0}\right)$ [2]. Models with $N$ doublets, which we shall call 'multi-Higgs-doublet models' (MHDM) are possible [3], and in particular two doublets are required for the minimal Supersymmetric Standard Model (MSSM) 四. One of the main goals at LEP2 will be to search for the above Higgs bosons, with previous searches at LEP having produced bounds of $M_{\phi^{0}} \geq 65.1 \mathrm{GeV}$ [5] for the minimal SM, and $M_{h^{0}} \geq 44 \mathrm{GeV}$ [6] for the lightest CP-even neutral Higgs boson $\left(h^{0}\right)$ of the MSSM.

Although there are many theoretically sound extended Higgs models, most attention is given to the minimal SM and the MSSM. In this paper we study the various non-supersymmetric non-minimal Higgs models in order to ascertain whether distinctive signatures are possible. We shall focus on the lightest CP-even neutral scalar $\left(h_{1}\right)$ and its phenomenology at LEP2, assuming that this Higgs particle is the only one in range at this collider. If a neutral Higgs boson is detected at LEP2 it is important to know from which model it originates. We shall only consider models with Higgs isospin doublets and our work is organized as follows. In Section 2 we introduce the relevant non-minimal Higgs models and investigate their couplings. Section 3 examines their phenomenology at LEP2 in the case of distinctive branching ratios not possible for $\phi^{0}$ and $h^{0}$. Finally, Section 4 contains our conclusions.

\section{Extended Higgs Sectors}

The minimal SM consists of one Higgs doublet $(T=1 / 2, Y=1)$, although extended sectors can be considered and have received substantial attention in the literature. For a general review see Ref. [4. There are two main constraints on such models:

(i) There must be an absence of flavour changing neutral currents (FCNC).

(ii) The rho parameter, $\rho=M_{W}^{2} /\left(M_{Z}^{2} \cos ^{2} \theta_{W}\right)$, must be very close to one.

Condition (i) is satisfied by constraining the Yukawa couplings to the fermions [7]. Condition (ii) requires models with only doublets, to which any number of singlets $(T=0, Y=0)$ can be added. Models with triplets $(T=1)$ [8], [9], [10] can also be considered, although obtaining $\rho \approx 1$ is achieved in a less natural way than for cases with only doublets.

The theoretical structure of the two-Higgs-doublet model (2HDM) is well known [4], while the general multi-Higgs-doublet model (MHDM) [3], 11], [12] has received substantially less attention. In particular when the MHDM is considered in the literature the focus is nearly always on the charged Higgs sector. We shall be investigating 
the properties of the lightest neutral CP-even Higgs boson of the MHDM, and for simplicity assume no mixing between CP-even and CP-odd scalars. It has been shown [3] that significant differences can exist between the 2HDM and MHDM in the case of the charged Higgs sector, and we anticipate an analogous result in the neutral sector.

\begin{tabular}{|c|c|c|c|c|}
\hline & Model I & Model I' & Model II & Model II' \\
\hline $\mathrm{u}$ (up-type quarks) & 2 & 2 & 2 & 2 \\
\hline $\mathrm{d}$ (down-type quarks) & 2 & 2 & 1 & 1 \\
\hline $\mathrm{e}$ (charged leptons) & 2 & 1 & 1 & 2 \\
\hline
\end{tabular}

Table 1: The four distinct structures of the 2HDM.

Table 1 shows the four different ways with which the 2HDM can be coupled to the fermions (the Yukawa couplings). The numbers (1 or 2) show which Higgs doublet couples to which fermion type. The Higgs sector of the MSSM requires Model II type couplings and thus the phenomenology of Model II has received the most attention of the four. Models $\mathrm{I}^{\prime}$ and $\mathrm{II}^{\prime}$ are rarely mentioned, and only then in the context of the charged Higgs sector [13]. For a MHDM there are more permutations of the Yukawa couplings, although it is conventional to couple each fermion type to a distinct doublet, e.g. in a 3 HDM doublets $1,2,3$ would couple to $\mathrm{d}$, $\mathrm{u}$, e respectively. We shall be considering the lightest CP-even Higgs scalar $\left(h_{1}\right)$ of the above models, and for the 2HDM its couplings to the fermions are given in Table 2.2

\begin{tabular}{|c|c|c|c|c|}
\hline & Model I & Model I' & Model II & Model II $^{\prime}$ \\
\hline$h u \bar{u}$ & $\cos \alpha / \sin \beta$ & $\cos \alpha / \sin \beta$ & $\cos \alpha / \sin \beta$ & $\cos \alpha / \sin \beta$ \\
\hline$h \bar{d}$ & $\cos \alpha / \sin \beta$ & $\cos \alpha / \sin \beta$ & $-\sin \alpha / \cos \beta$ & $-\sin \alpha / \cos \beta$ \\
\hline$h e \bar{e}$ & $\cos \alpha / \sin \beta$ & $-\sin \alpha / \cos \beta$ & $-\sin \alpha / \cos \beta$ & $\cos \alpha / \sin \beta$ \\
\hline
\end{tabular}

Table 2: The fermion couplings of $h_{1}$ in the 2HDM relative to those for the minimal SM Higgs boson $\left(\phi^{0}\right)$.

Here $\alpha$ is a mixing angle used to diagonalize the $\mathrm{CP}$-even mass matrix and $\beta$ is defined by $\tan \beta=v_{2} / v_{1}\left(v_{i}\right.$ is the VEV of the $i^{\text {th }}$ doublet and $\left.v_{1}^{2}+v_{2}^{2}=246^{2} \mathrm{GeV}^{2}\right)$. In the MSSM, which is a constrained version of the $2 \mathrm{HDM}$ (Model II), the angles $\alpha$ and $\beta$ are related. For the models that we shall consider $\alpha$ and $\beta$ are independent. There exists a bound on $\tan \beta$ of

$$
\tan \beta \geq 1.25
$$

obtained from considering the effects of the charged Higgs scalar on the $Z \rightarrow b \bar{b}$ decay [3].

\footnotetext{
${ }^{2}$ For Higgs masses in the range of LEP2, branching ratios to vector bosons are negligible.
} 
For the MHDM there exist $N$ doublets with the VEVs $\left(v_{i}\right)$ obeying the relationship

$$
v^{2}=\sum_{i=1}^{N} v_{i}^{2} .
$$

The lightest $\mathrm{CP}$-even mass eigenstate is a linear combination of the Lagrangian eigenstates, and can be written as

$$
h_{1}=\sum_{i=1}^{N} X_{i 1}^{*} \phi_{i}^{0} .
$$

The parameters $X_{i 1}^{*}$ originate from the mixing matrix for the CP-even neutral Higgs sector defined by

$$
\phi_{i}^{0}=\sum_{j=1}^{N} X_{i j} h_{j} .
$$

They are therefore analogous to $\sin \alpha$ and $\cos \alpha$ in the $2 \mathrm{HDM}$. Our convention is that $\phi_{i}^{0}$ is the real part of the neutral Higgs field of the $i^{\text {th }}$ doublet; $h_{j}$ are the mass eigenstates with $h_{1}$ taken to be the lightest. The unitarity of the matrix forces the relationship

$$
\sum_{i=1}^{N}\left|X_{i 1}\right|^{2}=1 .
$$

The Yukawa couplings in the MHDM are not as correlated as in the 2HDM. Therefore a MHDM can always feign a 2HDM but might possibly possess a distinctive signature. We shall now quantify the above statement. In the 2HDM there exists the relation $v^{2}=v_{1}^{2}+v_{2}^{2}$. Since the strength of a Yukawa coupling is proportional to $v / v_{i}$, for small $v_{i}$ the coupling is significantly enhanced relative to the minimal SM Higgs boson $\left(\phi^{0}\right)$. We see that if $v_{1}$ (say) is small, then $v_{2} \approx v$ is automatic. Therefore any Yukawa coupling which is proportional to $v_{2}$ cannot be enhanced significantly compared to $\phi^{0}$. However in the MHDM one has the relation in Eq. (2). This is a weaker constraint on the $v_{i}$ and therefore it is entirely possible that several $v_{i}$ are simultaneously small and hence a significantly different phenomenology may be possible. The Yukawa couplings for a MHDM are of the form

$$
v X_{i 1}^{*} / v_{i}
$$

and so the enhancements possible in the 2HDM are attainable if $v_{i} \ll v$.

We must next study the branching ratios (BRs) of the 2HDM and MHDM with the aim of obtaining distinct signatures not possible for $\phi^{0}$ or $h^{0}$ of the MSSM. For Higgs masses in the range of LEP2 $\left(M_{\phi^{0}} \leq 100 \mathrm{GeV}\right)$ the only important decays are to $b \bar{b}, \tau^{+} \tau^{-}, c \bar{c}$ and $g g_{\text {. For }} \phi^{0}$ with $M_{\phi^{0}}=50 \mathrm{GeV}$ the ratios of the respective BRs are approximately [14]

$$
b \bar{b}: \tau^{+} \tau^{-}: c \bar{c}: g g=0.87: 0.08: 0.03: 0.02 .
$$

\footnotetext{
${ }^{3} \mathrm{QCD}$ corrections are quite sizeable and have been included.
} 
For $M_{\phi^{0}}=100 \mathrm{GeV}$ one has:

$$
b \bar{b}: \tau^{+} \tau^{-}: c \bar{c}: g g=0.80: 0.09: 0.03: 0.07 .
$$

Here the BRs for $b \bar{b}$ and $c \bar{c}$ are evaluated by summing the Feynman diagrams in Figure 1 , with the replacements $q=b$ and $q=c$ respectively. The virtual QCD corrections have not been displayed in the figures although they are included in Eqs. (7) and (8). The first two diagrams give the dominant contributions [15]. The BR for $g g$ represents the summation of all diagrams which give light quark jets i.e. all diagrams in Figure 1 with $q=u, d, s$, and both diagrams in Figure 2. We note that decays via a quark loop proceed predominantly by a top quark loop.
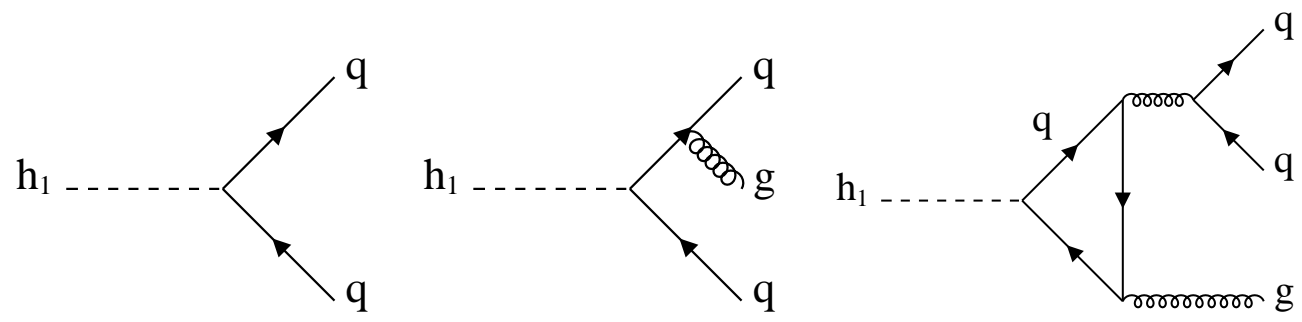

Figure 1: Decays of $h_{1} \rightarrow q \bar{q}, q \bar{q} g$.
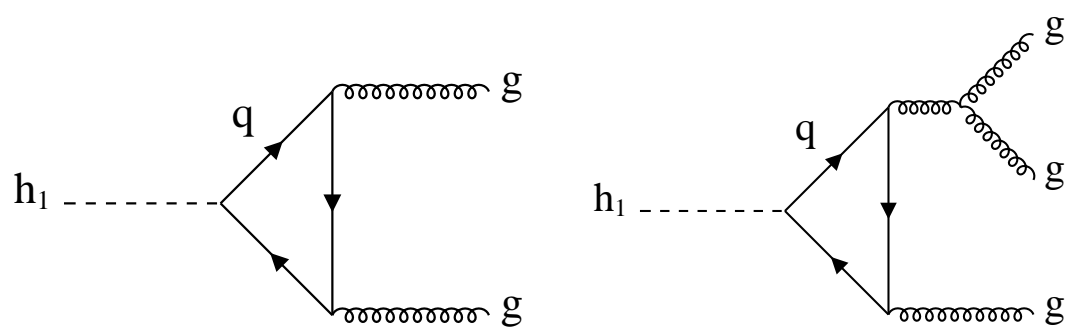

Figure 2: Decays of $h_{1} \rightarrow g g(g)$.

In Eq. (8) decays to $W W^{*}$ (where * denotes an off-shell particle) are at the $1 \%$ level. It is apparent from Eqs. (7) and (8) that decays to $b \bar{b}$ dominate, and this property allows light quark backgrounds to the Higgs signal to be reduced by $b$-tagging. For $h^{0}$ one has almost exactly the same BRs, although the $g g$ and $c \bar{c}$ decays are usually $\leq 1 \%$ unless $\tan \beta$ is large [14]; for large $\tan \beta$ they are both of the order $5 \%$. Distinguishing $\phi^{0}$ from $h^{0}$ provides a challenge for future colliders and is discussed elsewhere [16]. It is the aim of this paper to study the phenomenology of the $2 \mathrm{HDM}$ and MHDM 
at LEP2, in the case of extreme BRs not possible for $\phi^{0}$ and $h^{0}$. From Table 2 one can vary the angles $\alpha$ and $\beta$ independently to see if parameter spaces exist for such extreme BRs. We can summarize as follows:

(i) Model I': $\mathrm{BR}\left(h_{1} \rightarrow \tau^{+} \tau^{-}\right)$large if $\tan \beta \gg 1$ and $\sin \alpha$ moderate 4 to maximal.

(ii) Model II': $\mathrm{BR}\left(h_{1} \rightarrow \tau^{+} \tau^{-}\right)$, BR $\left(h_{1} \rightarrow c \bar{c}\right)$ and $\mathrm{BR}\left(h_{1} \rightarrow g g\right)$ share domination for small $\sin \alpha$ and $\tan \beta$ moderate.

(iii) Model II: $\mathrm{BR}\left(h_{1} \rightarrow c \bar{c}\right)$ and $\mathrm{BR}\left(h_{1} \rightarrow g g\right)$ share domination for small $\sin \alpha$ and $\tan \beta$ moderate.

(iv) Model I: Has exactly the same BRs as $\phi^{0}$.

One must be aware of the bound $\tan \beta \geq 1.25$ from Eq. (1) when varying the angles. It is apparent that distinctive BRs are possible in the $2 \mathrm{HDM}$, the general requirement being that one of the angles has an extreme value. Most noticeably the $b \bar{b}$ decays can be suppressed and/or made negligible 3 . This is in contrast to $\phi^{0}$ and $h^{0}$ which will decay predominantly to $b \bar{b}$ for masses in range at LEP2 - see Eqs. (7) and (8). It is our aim to exploit these enhanced BRs at LEP2. On first viewing it seems that $\mathrm{BR}\left(h_{1} \rightarrow \tau^{+} \tau^{-}\right) \gg 10 \%$ would provide a clear signal of Model $\mathrm{I}^{\prime}$, since searching for $\phi^{0} \rightarrow \tau^{+} \tau^{-}$in conjunction with $Z \rightarrow$ hadrons is one of the proposed techniques at LEP2 and gives a reasonable signal [14. However, the production cross-section $\sigma\left(e^{+} e^{-} \rightarrow Z^{*} \rightarrow Z h_{1}\right)$ is suppressed relative to that of $\phi^{0}$ by a factor of $\sin ^{2}(\beta-\alpha)$ [4]. Therefore we must investigate whether the parameter choices needed to obtain a distinctive BR simultaneously decrease the production cross-section at LEP2. We shall address this problem in the next section, as well as applying the proposed search techniques for $\phi^{0}$ to the 2HDM and MHDM.

\section{Phenomenology at LEP2}

The phenomenology of $\phi^{0}$ at LEP2 has been extensively covered in the literature, and recently in Ref. [14]. We shall be applying these various search techniques for the $2 \mathrm{HDM}$ and MHDM, and so will now give a short review of them.

The dominant production process for $\phi^{0}$ if $M_{\phi^{0}}$ is in the range of LEP2 $\left(M_{\phi^{0}} \leq\right.$ $\left.\sqrt{s}-M_{Z} \mathrm{GeV}\right)$ is the Higgs-strahlung process [17]

$$
e^{+} e^{-} \rightarrow Z \phi^{0}
$$

\footnotetext{
${ }^{4}$ We take 'moderate' to mean the angle is approximately $\pi / 4$.

${ }^{5}$ We note that the width of the third diagram in Figure 1 (with $q=b$ ) would be boosted if the coupling to up-type quarks is enhanced. However, this diagram has a very small width [15] and so heavily suppressed $h_{1} \rightarrow b \bar{b}$ decays are still possible.
} 
in which $\phi^{0}$ is emitted from a virtual $Z$ boson. This cross-section is well known and includes various loop corrections. We shall be interested in the case of $\sqrt{s}=192$ $\mathrm{GeV}$, since it is at this energy that the computer simulations in Ref. [14] have been carried out. The cross-section for $\phi^{0}$ is $\sigma=0.95(0.40) \mathrm{pb}$ if $M_{\phi^{0}}=60(90) \mathrm{GeV}$. For $M_{\phi^{0}}=100 \mathrm{GeV}$, which is the mass generally considered to be at the limit of the LEP2 range for $\sqrt{s}=192 \mathrm{GeV}, \sigma=0.1 \mathrm{pb}$. Experimental simulations have been performed for the following event types:

(i) $Z \rightarrow e^{+} e^{-} ; \mu^{+} \mu^{-}, \phi^{0} \rightarrow$ anything.

(ii) $Z \rightarrow \tau^{+} \tau^{-}, \phi^{0} \rightarrow$ hadrons and vice versa.

(iii) $Z \rightarrow \nu \bar{\nu}, \phi^{0} \rightarrow$ hadrons.

(iv) $Z \rightarrow$ hadrons, $\phi^{0} \rightarrow b \bar{b}$.

Events (iii) and (iv) require $b$-tagging to reduce the light quark background, and since we shall be considering Higgs bosons with suppressed $b \bar{b}$ decays these will not be relevant to our analysis. Events of the form (i) are independent of the Higgs BRs, and require a pair of energetic leptons with an invariant mass compatible with $M_{Z}$. The Higgs signal appears as a peak in the missing mass distribution. Events of the form (ii) are characterized by two energetic, isolated $\tau$ 's associated with two hadronic jets. The existence of a Higgs boson would then be observed as an accumulation around $\left(M_{\phi^{0}}, M_{Z}\right)$ in the invariant mass distributions of the $\tau$ 's and jets. Table 3 (from Ref. [14]) shows the simulated effective cross-section in the four channels for $M_{\phi^{0}}=90$ $\mathrm{GeV}$ and $\sqrt{s}=192 \mathrm{GeV}$ :

\begin{tabular}{|c|c|c|c|c|}
\hline & $\phi^{0} l^{+} l^{-}$ & $\tau^{+} \tau^{-} q \bar{q}$ & $\phi^{0} \nu \bar{\nu}$ & $\phi^{0} q \bar{q}$ \\
\hline ALEPH & $12: 12$ & $8: 5$ & $24: 13$ & $58: 33$ \\
\hline DELPHI & $6: 2$ & $4: 6$ & $25: 18$ & $46: 36$ \\
\hline L3 & $13: 7$ & - & $28: 21$ & $36: 23$ \\
\hline OPAL & $8: 30$ & - & $17: 13$ & $34: 36$ \\
\hline
\end{tabular}

Table 3: Effective cross-sections for signal (first number) and background (second number) in fb, at LEP2 for $M_{\phi^{0}}=90 \mathrm{GeV}$ and $\sqrt{s}=192 \mathrm{GeV}$, taking into account acceptances and efficiencies (from Ref. [14]).

All the above analysis has been for $\phi^{0}$, with BRs given by Eqs. (7) and (8). It is now our aim to extrapolate these results for use in the 2HDM and MHDM. As mentioned in Section 2, these models (with the exception of Model I) may possess vastly different BRs to those of $\phi^{0}$. However, the production cross-section for $h_{1}$ is suppressed relative to that for $\phi^{0}$ (denoted by $\sigma_{\phi^{0}}$ ) by $\sin ^{2}(\beta-\alpha$ ). A possible consequence of this suppression is that a very light $h_{1}\left(M_{h_{1}} \approx 10 \mathrm{GeV}\right)$ would have 
escaped detection at LEP1 if $\sin ^{2}(\beta-\alpha) \ll 0.1$. This possibility has recently been considered in Refs. [18], [19 and these papers consider making use of non-suppressed production channels. For $h^{0}$ of the MSSM, $\sin ^{2}(\beta-\alpha)$ is close to one over essentially all of the available parameter space and so there exists the lower bound $M_{h^{0}} \geq 44 \mathrm{GeV}$ [6]. Therefore the detection of a neutral CP-even Higgs boson with a mass between $10 \mathrm{GeV}$ and $40 \mathrm{GeV}$ would indicate $h_{1}$. However, it would be impossible to infer from which specific model it originates unless it displays a distinctive BR.

To start our analysis let us now take the case of Model $\mathrm{I}^{\prime}$ with a dominant BR $\left(h_{1} \rightarrow\right.$ $\tau^{+} \tau^{-}$). One would therefore use detection channel (ii), and the event cross-section (before applying efficiencies and acceptances) would be given by $\sigma_{\phi^{0}} \times \sin ^{2}(\beta-\alpha)$ multipied by:

$$
\left[\mathrm{BR}(Z \rightarrow q \bar{q}) \times \mathrm{BR}\left(h_{1} \rightarrow \tau^{+} \tau^{-}\right)+\mathrm{BR}\left(h_{1} \rightarrow q \bar{q}\right) \times \mathrm{BR}\left(Z \rightarrow \tau^{+} \tau^{-}\right)\right] .
$$

Eq. (10) shows that the dominant term is the first since BR $(Z \rightarrow q \bar{q})=70 \%$, while $\mathrm{BR}\left(Z \rightarrow \tau^{+} \tau^{-}\right)=3.4 \%$. Now for $\phi^{0}$ we have the cross-section equal to

$$
0.086 \times \sigma_{\phi^{0}}
$$

For Model I' this may be greatly enhanced, e.g. for BR $\left(h_{1} \rightarrow \tau^{+} \tau^{-}\right)=80 \%$ and $\mathrm{BR}\left(h^{0} \rightarrow\right.$ jets $)=20 \%$ we have the following cross-section:

$$
0.57 \times \sigma_{\phi^{0}} \times \sin ^{2}(\beta-\alpha)
$$

Hence there could be a considerable increase in the signal size for this channel, as much as an order of magnitude. It is now necessary to see if the values of $\beta$ and $\alpha$ needed to give a large $\mathrm{BR}\left(h_{1} \rightarrow \tau^{+} \tau^{-}\right)$cause a suppression in the $\sin ^{2}(\beta-\alpha)$ term. We note from the couplings in Table 2 that $\alpha \rightarrow \pi / 2$ would further enhance $\tau^{+} \tau^{-}$ decays while suppressing the $b \bar{b}$ channel. However, from Eq. (12) we see that allowing both $\alpha \rightarrow \pi / 2$ and $\beta \rightarrow \pi / 2$ would cause strong suppression from $\sin ^{2}(\beta-\alpha)$ in the cross-section. We shall now derive the value of $\alpha$ that maximizes the number of events.

To obtain a large $\operatorname{BR}\left(h_{1} \rightarrow \tau^{+} \tau^{-}\right)$we showed in Section 2 that $\tan \beta$ must be large and $\sin \alpha$ must be moderate to maximal. In Eq. (10) the dominant term is the first one and so we shall neglect the second. The first term is proportional to $\sin ^{2} \alpha / \cos ^{2} \beta$, and so the number of events in this channel depends on $f(\beta, \alpha)$ defined by:

$$
f(\beta, \alpha)=\sin ^{2}(\beta-\alpha) \times \sin ^{2} \alpha / \cos ^{2} \beta .
$$

In Figure 3 we plot $f(\beta, \alpha)$ with $\beta=1.5 \operatorname{rads}\left(86^{\circ}\right)$ since large $\operatorname{BR}\left(h_{1} \rightarrow \tau^{+} \tau^{-}\right)$ requires small $\cos \beta$.

It is apparent from Figure 3 that moderate values of $\alpha(\approx \pi / 4)$ allow the largest values of $f(\beta, \alpha)$. We see that $\alpha=\pi / 4$ and $\beta \approx \pi / 2$ would give $\sin ^{2}(\beta-\alpha)=0.5$. 


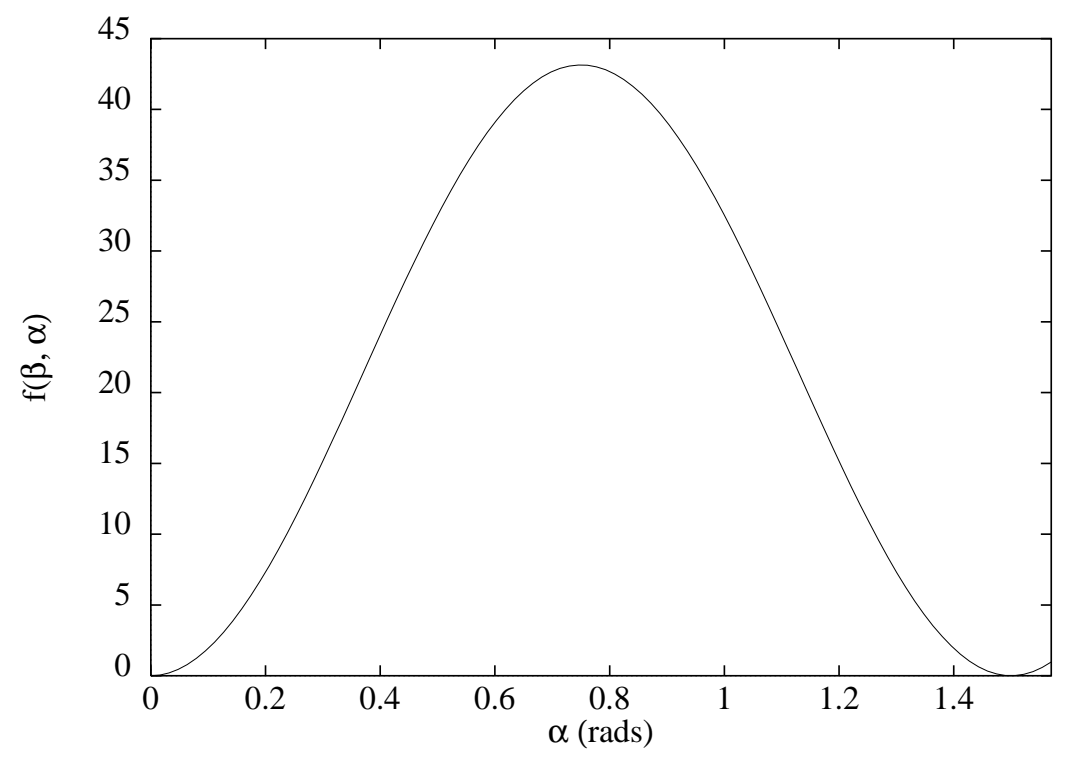

Figure 3: $f(\beta, \alpha)$ as a function of $\alpha$ for $\beta=1.5$ rads.

Therefore the suppression of the cross-section in Eq. (12) is not so severe, and the distinctive signature of a large number of $\tau^{+} \tau^{-}$plus 2 jet events is certainly possible in Model I'. Moreover, a large $\tau^{+} \tau^{-}$peak will be centered at $M_{h}$ with almost no peak at $M_{Z}$, which is in contrast to the $\phi^{0}$ case which will have small $\tau^{+} \tau^{-}$peaks centered at $M_{\phi}$ and $M_{Z}$ (since the two terms in Eq. (10) are of roughly equal magnitude). Hence, even if there is strong suppression from the $\sin ^{2}(\beta-\alpha)$ term, the relative heights of the peaks will be different for $h_{1}$. However there may not be enough events to notice this pattern unless $M_{h_{1}}$ is considerably lighter than $90 \mathrm{GeV}$, i.e. when the cross-section is larger. Table 3 shows that for the ALEPH detector with $500 \mathrm{pb}^{-1}$ of integrated luminosity one would expect the number of signal (background) events to be 4 (2). This is for $\phi^{0}$ with $M_{\phi}=90 \mathrm{GeV}$. For $M_{h_{1}}=60 \mathrm{GeV}$ (say) the improved cross-section would double the number of events.

For $h_{1}$ of Model I, Table 2 shows that its couplings to the fermions are identical to those of $\phi^{0}$ and hence no distinct signature is possible. We recall that $h^{0}$ of the MSSM also feigns $\phi^{0}$ over a large parameter space, but $h_{1}$ of Model I could have a suppressed production rate due to the factor $\sin ^{2}(\beta-\alpha)$; hence a neutral $\mathrm{CP}-$ even Higgs boson with identical BRs to $\phi^{0}$ but a suppressed cross-section and/or $M_{h_{1}} \leq 44$ $\mathrm{GeV}$ would be indirect evidence 6 for Model $\mathrm{I}^{\prime}$. Our earlier work shows that alternative

\footnotetext{
${ }^{6}$ Of course, another $2 \mathrm{HDM}$ or a MHDM could mimic the BRs of $\phi^{0}$ for carefully chosen couplings.
} 
distinctive signatures of Model I would be a fermiophobic Higgs [20] and/or a light charged scalar $\left(M_{H^{ \pm}} \leq 80 \mathrm{GeV}\right)$ [12].

For Model II' we stated in Section 2 that the decays $h_{1} \rightarrow \tau^{+} \tau^{-}, h_{1} \rightarrow g g$ and $h_{1} \rightarrow c \bar{c}$ would share the domination for small $\sin \alpha$ and $\tan \beta$ moderate, i.e. $h_{1} \rightarrow b \bar{b}$ may be heavily suppressed. The decay $h_{1} \rightarrow g g$ proceeds via a top quark loop and so is boosted since the above parameter choices enhance couplings to up-type quarks. From Eqs. (7) and (8) we see that the ratio of the $\tau^{+} \tau^{-}$partial width to the jet ( $c \bar{c}$ and $g g$ ) partial width would be 8:5 for $M_{h_{1}}=50 \mathrm{GeV}$, and 9:10 for $M_{h_{1}}=100$ $\mathrm{GeV}$. Therefore in contrast to Model $\mathrm{I}^{\prime}$ the $\tau^{+} \tau^{-}$decays can never saturate the total width (i.e. $\mathrm{BR} \approx 100 \%$ ). Hence it would appear that Model II' could be mimicked by Model I', although in this case Model $\mathrm{I}^{\prime}$ would have accompanying $b \bar{b}$ decays while Model II' would be accompanied by $c \bar{c}$ and $g g$. If the $b \bar{b}$ decays are of sufficient magnitude then Model $\mathrm{I}^{\prime}$ would also register a signal in detection methods (iii) and (iv) (which require $b$-tagging), while Model II' would not. Therefore it is possible that $h_{1}$ of Model II' has a distinct signature.

Model II is the structure that is most considered in the literature since it is the form of the MSSM. As shown in Section 2 for suitable parameter choices the $b \bar{b}$ channel can be suppressed and the decays $h_{1} \rightarrow g g$ and $h_{1} \rightarrow c \bar{c}$ would dominate. We do not believe that this scenario has been considered before, and any detection technique that requires $b$-tagging or $\tau^{+} \tau^{-}$decays will fail. However, the missing mass technique is Higgs BR independent and so there is still a chance of a signal, provided that the cross-section suppression is not too great. Figure 4 plots the suppression $\sin ^{2}(\beta-\alpha)$ as a function of $\alpha$ for $\beta=\pi / 4$ (moderate $\tan \beta$ is required for an enhancement of $c \bar{c}$ and $g g$ decays) and shows that for very small $\alpha$ (i.e. $\sin \alpha \approx 0$, which is of interest to us) the cross-section suppression is only of order 0.5. Therefore we anticipate that a reasonable signal could be obtained for the scenario of $c \bar{c}$ and $g g$ decays dominating; see Table 3 for an indication of event numbers. We stress that the non-observance of a Higgs signal in the other three detection channels (which make use of $b \bar{b}$ and $\tau^{+} \tau^{-}$ decays), but a positive signal using the missing mass technique would be evidence for $h_{1}$ of Model II with $h_{1} \rightarrow c \bar{c}, g g$ decays dominating. Moreover, if such a signal is observed there would then be motivation to search for charm rich jets originating from $h_{1}$. We conclude that Model II does possess a distinctive signature.

Having completed our account of the 2HDM at LEP2 we now address the MHDM. As mentioned earlier, a MHDM can always feign a $2 \mathrm{HDM}$ but could it also possess a distinctive signature? Concerning decay channels, the 2HDM can exhibit extreme BRs for the decays to up-type quarks, down-type quarks and leptons. Of course the MHDM can possess identical enhancements (Eq. (6)) but this is not distinctive. However, if the production cross-section of $h_{1}$ from the MHDM has less suppression than $h_{1}$ from the 2HDM, as well as having an extreme BR, then this would be a distinguishing trait. The coupling of the MHDM neutral scalars to $Z$ (which is $\sin ^{2}(\beta-\alpha)$ 


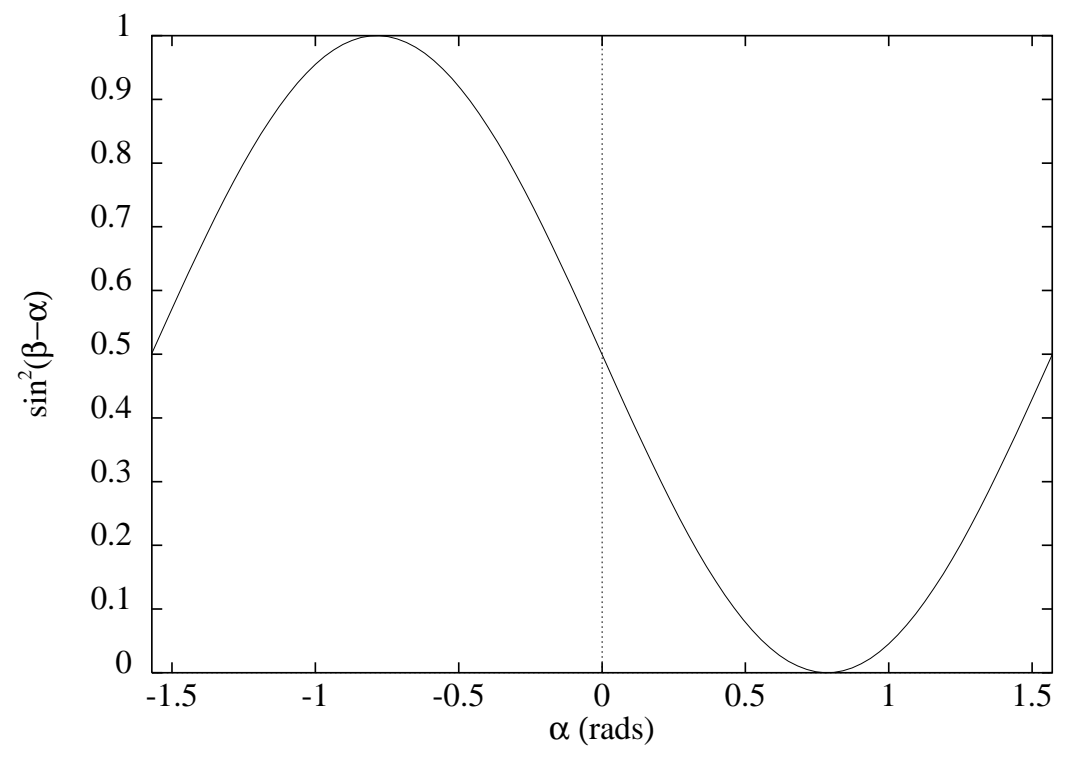

Figure 4: $\sin ^{2}(\beta-\alpha)$ as a function of $\alpha$ for $\beta=\pi / 4$ rads.

in the $2 \mathrm{HDM}$ ) is given by

$$
\frac{\sigma_{h_{1}}}{\sigma_{\phi^{0}}}=\frac{\left|\sum v_{i} X_{i 1}\right|^{2}}{v^{2}} .
$$

We recall that the scenario of an extreme $\mathrm{BR}$ in the $2 \mathrm{HDM}$ is accompanied by a cross-section suppression of $\leq 0.5$. From Eq. (14) we can show that the suppression in the MHDM could be less. This is the case if a particular $v_{i}^{2}$ is much larger than the others $\left(v_{i}^{2} \rightarrow 246^{2} \mathrm{GeV}^{2}\right)$, as well as possessing $\left|X_{i 1}^{2}\right|$ close to 1 . Therefore there exist parameter spaces for

$$
0.5 \leq \frac{\sigma_{h_{1}}}{\sigma_{\phi^{0}}} \leq 1
$$

\section{Conclusions}

We have studied the phenomenology of the lightest CP-even neutral Higgs boson $\left(h_{1}\right)$ of a non-SUSY, non-minimal Standard Model at LEP2. We considered the four versions of the $2 \mathrm{HDM}$ and a general MHDM, and showed that $h_{1}$ could possess branching ratios (BRs) significantly different to those of the minimal Standard Model Higgs boson $\left(\phi^{0}\right)$ and $h^{0}$ of the Minimal Supersymmetric Standard Model; $\phi^{0}$ and $h^{0}$ decay predominantly to $b \bar{b}$ for masses in the range of LEP2. It is assumed that $h_{1}$ is the only Higgs boson in range at LEP2. The production cross-section of $e^{+} e^{-} \rightarrow Z h_{1}$ is 
suppressed relative to that of $\phi^{0}$ by a factor $\sin ^{2}(\beta-\alpha)$ in the $2 \mathrm{HDM}$ and an analogous factor in the MHDM; a consequence of this suppression is that a very light $h_{1}$ with $10 \mathrm{GeV} \leq M_{h_{1}} \leq 44 \mathrm{GeV}$ may have escaped detection at LEP1. We showed that Model I' may exhibit a large BR $\left(h_{1} \rightarrow \tau^{+} \tau^{-}\right)$which would register a clear signal. Model II may decay predominantly to $c \bar{c}$ and $g g$; this would provide a distinctive signature since it could only be observed as missing mass recoiling against a lepton pair (from $Z \rightarrow l^{+} l^{-}$), with no signal in the other detection channels. For Model I, $h_{1}$ possesses the same BRs as $\phi^{0}$ and so does not have a distinctive signature. Model II' may decay predominantly to a mixture of $\tau^{+} \tau^{-}, c \bar{c}$ and $g g$; the enhanced $\tau^{+} \tau^{-}$channel could be mimicked by Model $\mathrm{I}^{\prime}$, but these latter decays would be accompanied by $b$ quark jets, in contrast to the light quark/charm rich jets which would accompany the enhanced $\tau^{+} \tau^{-}$channel in Model $\mathrm{II}^{\prime}$. Therefore it is possible that Model $\mathrm{II}^{\prime}$ possesses a distinct signature. In the MHDM $h_{1}$ can feign any of the above extreme BRs but may be distinguished from $h_{1}$ of the $2 \mathrm{HDM}$ if its production cross-section is close to that of $\phi^{0}$.

\section{Acknowledgements}

I wish to thank W.J. Stirling and C.J. Dove for reading the manuscript. This work has been supported by the UK PPARC.

\section{References}

[1] S. Weinberg, Phys. Rev. Lett. 19 (1967) 1264.

[2] P.W. Higgs, Phys. Lett. B12 (1964) 132, Phys. Rev. Lett. 13 (1964) 508.

[3] Y. Grossman, Nucl. Phys. B426 (1994) 355.

[4] J.F. Gunion, H.E. Haber, G.L. Kane and S. Dawson, The Higgs Hunter's Guide (Addison-Wesley, Reading, 1990).

[5] A. Sopczak, preprint CERN-PPE/95-46 (1995).

[6] P. Abreu et al., Delphi Collaboration CERN-PPE/94-218 (1994).

[7] S.L. Glashow and S. Weinberg, Phys. Rev. D15 (1977) 1958.

[8] H. Georgi and M. Machacek, Nucl. Phys. B262 (1985) 463.

[9] J.F. Gunion, R. Vega, and J. Wudka, Phys. Rev. D42 (1990) 1673; Phys. Rev. D43 (1991) 2322. 
[10] A.G. Akeroyd, Phys. Lett. B353 (1995) 519.

[11] G.C. Branco, A.J. Buras and J.M. Gerard, Nucl. Phys. B259 (1985) 306.

[12] A.G. Akeroyd and W.J. Stirling, Nucl. Phys. B447 (1995) 3.

[13] V. Barger, J.L. Hewett and R.J.N. Phillips, Phys. Rev. D41 (1990) 3421.

[14] LEP2 Interim Report, preprint CERN-TH/95-151, CERN-PPE/95-78 (1995) and references therein.

[15] A. Djouadi, M. Spira and P.M. Zerwas, preprint DESY 95-210, (1995).

[16] H. E. Haber, preprint CERN-TH/95-109, SCIPP 95/15, (1995) and references therein.

[17] J. Ellis, M.K. Gaillard, and D.V. Nanopoulos, Nucl. Phys. B106 (1976) 292; B.L. Ioffe and V.A. Khoze, Sov. Journal of Phys. 9 (1978) 50; B.W. Lee, C. Quigg, and H.B. Thacker, Phys. Rev. D16 (1977) 1519; J.D. Bjorken, Proc. Summer Institute on Particle Physics, SLAC Report 198 (1976).

[18] T.V. Duong, E. Keith, E. Ma and H. Kikuchi, Phys. Rev. D52 (1995) 5045.

[19] J. Kalinowski and M. Krawczyk, Phys. Lett. B361 (1995) 66.

[20] A.G. Akeroyd, Phys. Lett. B368 (1996) 89 . 